\title{
The influence of Nordic walking on isokinetic trunk muscle endurance and sagittal spinal curvatures in women after breast cancer treatment
}

\author{
JuSTYNA MARIA HANUSZKIEWICZ*, MAREK WoŹNIEWSKI, IWONA MALICKA \\ Department of Rehabilitation in Internal Diseases, Faculty of Physiotherapy, \\ University School of Physical Education in Wrocław, Wrocław, Poland.
}

\begin{abstract}
Purpose: The aim of this work was to assess the impact of Nordic walking on sagittal spinal curvatures and isokinetic trunk muscle endurance in women after breast cancer treatment. Methods: Thirty-nine breast cancer survivors were divided into two groups: a study group $(n=19)$ that performed Nordic walking, and a control group $(n=20)$ that performed a standard general exercise programme. Body posture was assessed using Moiré photogrammetry and trunk muscle (flexors and extensors) endurance at $120^{\circ} / \mathrm{s}$ was measured isokinetically. Statistical analyses were based on two-way ANOVA with Scheffe post hoc tests and Pearson's $r$ correlation tests. Results: Women who completed the 8-week Nordic walking intervention showed significant improvements in average power and total work, irrespective of the muscle group investigated. Following training intervention, greater strength-velocity values of the trunk muscles were observed in the study group, compared to the control group. There were no significant changes in postural parameters or correlations for trunk muscle function within spinal curvatures before and after the training interventions. When both groups were combined (Nordic walking + general exercises), functional-postural correlations following the intervention showed a statistically significant tendency toward a reduced inclination of the upper thoracic section, together with increases in all tested functional parameters of the trunk flexor and extensor muscles $(r=-0.33$ to $r=-0.37)$. Conclusions: Compared to a standard general exercise programme, Nordic walking is more effective for improving isokinetic trunk muscle endurance in women after breast cancer. However, no changes in sagittal spinal curvatures were observed after the 8-week Nordic walking and general exercise interventions.
\end{abstract}

Key words: physical activity, Nordic walking, body posture, isokinetics, photogrammetry

\section{Introduction}

Nordic walking (NW) is a common gait-based activity based on a natural and at the same time simple movement of humans [16]. NW involves almost 90\% of all human muscles. The NW technique, which employs walking poles, activates the muscles of the shoulder girdle (particularly the anterior part of the deltoid muscle), upper limbs (mainly the triceps brachii and forearm muscles), back (latissimus dorsi and teres major muscles), chest and abdominal muscles (pectoralis major and oblique muscles). Although these muscles are, to a large extent, responsible for maintaining proper posture [15], [21], research on the muscular response for this exercise is generally limited to the upper and lower limbs [23]. The muscles of the trunk modulate the activity of the lower and upper limbs during walking; however, above all, they are of fundamental importance for maintaining body balance and spine stabilisation. The inclusion of poles when walking requires greater control of the trunk to extend the step length and balance the torque generated by the poles, which would otherwise rotate the upper

\footnotetext{
* Corresponding author: Justyna Maria Hanuszkiewicz, University School of Physical Education in Wrocław, I.J. Paderewskiego 35, 51-612 Wrocław, Poland. Phone: +48 713473519, e-mail: justyna.hanuszkiewicz@awf.wroc.pl

Received: February 26th, 2020

Accepted for publication: March 20th, 2020
} 
trunk along the longitudinal axis of the body [1], [22]. Furthermore, Aruin and Latash [1] demonstrated that arm swinging recruits several postural muscles to help maintain balance.

The process of maintaining correct body posture is conditioned by long-lasting muscular work and is considered an endurance activity. This is consistent with the work performed during NW. Walking with poles is a full-body exercise that simultaneously develops the upper and lower muscles of the body. This exercise also strengthens the paraspinal muscles as participants are required to maintain a steady position for a long period, achieved by simultaneous activation of the abdominal muscles, pelvic muscles and sensory nervous system. This exercise can improve muscle strength and muscle endurance, and, therefore, could be useful for recovering the normal angle balance of the trunk [16].

Breast cancer (BC), together with the adverse effects associated with treatment, can cause changes in body posture. Disorders of the musculoskeletal system may result from anatomical changes that occur in the armpit following surgery and motor dysfunctions of the upper limb and spine [14]. These changes affect body posture and the ability of the spine to carry loads, as well as its proper functioning [2], [10]. Moreover, significant changes in the anatomical structure of the trunk can also affect spatiotemporal gait parameters, such as the step length and walking velocity [7].

Nordic walking is widely used in the rehabilitation of patients undergoing cardiological, orthopaedic, neurological and oncological treatment. The safety and attractiveness of walking exercises with poles has been confirmed, along with improvements in the physical and psychological functioning of $\mathrm{BC}$ survivors [8], [12], [21]. However, previous studies on NW intervention have not evaluated the influence of body posture and spine kinetic disturbances on the dynamic parameters of gait. For this reason, we examined the effect of NW on trunk muscle function and spinal curvatures in the sagittal plane of women who had undergone treatment for $\mathrm{BC}$.

\section{Materials and methods}

\subsection{Participants}

To participate in the study, patients were qualified by a physiotherapist under the supervision of a physician. Initially, we recruited a sample of 58 potentially eligible respondents who met the following inclusion criteria: diagnosis of $\mathrm{BC}$ (with a radical or saving procedure), female gender, age between 50 and 75 years, and a period of at least 1 year since treatment. Participants were excluded if they had lymphedema of the upper extremities or comorbidities in the form of mental, orthopaedic, cardiological, neurological or oncological (cancers other than BC) illnesses. Following eligibility screening, the baseline sample included 39 patients.

Participants were divided into two groups. The study group (SG) performed NW $(n=19)$, while the control group (CG) performed a standard general exercise programme $(n=20)$. This was a randomised controlled trial, and participants were allocated into groups via sealed opaque envelopes.

Intergroup comparisons of somatic parameters and time since surgery showed no statistically significant differences (Table 1). Similarly, the analysis of treatment methods revealed no statistically significant differences between the groups (Table 2).

Table 1. Somatic parameters and time since treatment in the study and control groups

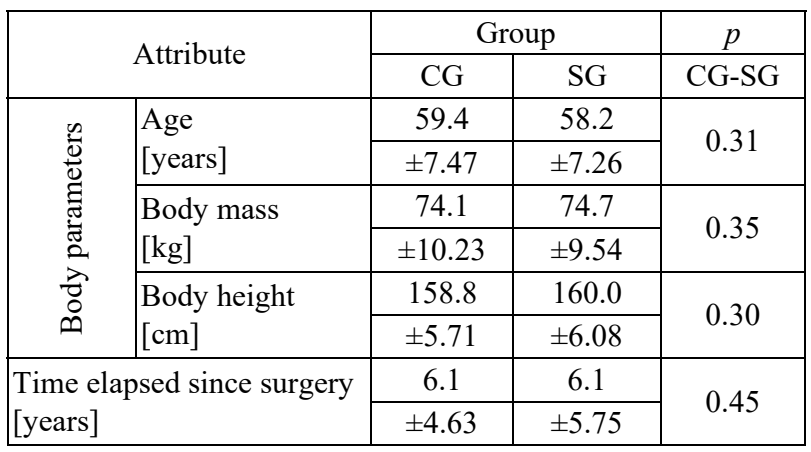

$\mathrm{CG}$ - control group, $\mathrm{SG}$ - study group, values are expressed as mean \pm standard deviation.

Table 2. Breast cancer treatment modalities for participants in the study and control groups

\begin{tabular}{|l|l|c|c|c|c|}
\hline \multirow{2}{*}{ Treatment modality } & \multicolumn{2}{|c|}{ Group } & \multirow{2}{*}{$\begin{array}{c}\chi^{2} \\
\text { Pearson }\end{array}$} & \multirow{2}{*}{$p$} \\
\cline { 3 - 6 } & CG & SG & \\
\hline \multirow{2}{*}{$\begin{array}{l}\text { Type } \\
\text { of procedure }\end{array}$} & Patey & $80 \%$ & $84 \%$ & 0.1173 & 0.73 \\
\hline \multirow{3}{*}{$\begin{array}{l}\text { Adjuvant } \\
\text { treatment }\end{array}$} & BCT & $20 \%$ & $16 \%$ & 0.1173 & 0.73 \\
\cline { 2 - 6 } & $\begin{array}{l}\text { Radiotherapy } \\
\text { therapy }\end{array}$ & $45 \%$ & $47 \%$ & 0.220 & 0.88 \\
\cline { 2 - 6 } & Chemotherapy & $70 \%$ & $74 \%$ & 0.6531 & 0.80 \\
\hline \multirow{2}{*}{$\begin{array}{l}\text { Operated } \\
\text { side }\end{array}$} & Right & $65 \%$ & $53 \%$ & 0.6160 & 0.43 \\
\cline { 2 - 6 } & Left & $50 \%$ & $58 \%$ & 0.2444 & 0.62 \\
\hline \multirow{2}{*}{ Mastectomy } & Single & $85 \%$ & $89 \%$ & 0.1745 & 0.68 \\
\cline { 2 - 6 } & Double & $15 \%$ & $11 \%$ & 0.1745 & 0.68 \\
\hline
\end{tabular}

CG - control group, SG - study group, BCT - breast conserving therapy. 
The study was approved by the Local Bioethics Committee of the University of Physical Education in Wroclaw. The participants received oral and written information about the study and signed an informed and voluntary consent for participation.

\subsection{Functional examination of the trunk muscles}

Assessment of trunk muscle function was performed at the Laboratory of Functional Studies using the Biodex Multi-Joint 3 Isokinetic Dynamometer. Measurements of alternating trunk flexion and extension with a maximum force in the shortest time at a selected angular velocity $\left(120^{\circ} / \mathrm{s}\right)$ were recorded, and the endurance characteristics were specified (20 repetitions).

The trunk and thighs were stabilised with straps attached to a chair to eliminate any supportive movements. The participant was placed in a semi-standing position in a chair so that the axis of rotation of the joint and the dynamometer were aligned. The pivot point was determined at L5-S1.

The same range of trunk flexion and extension was set around $70^{\circ}\left(20^{\circ}\right.$ extension, $50^{\circ}$ flexion $)$ for all participants. It was set up in such way that all participants could perform the same movement without pain, allowing standardisation of intergroup comparisons. Each measurement was preceded by a verbal instruction and warm-up exercise (several test runs).

The parameters total work - TW [J] and average power - AP [W] were selected for endurance measurements of the trunk muscles, based on existing examinations using isokinetic tests [3], [10], [18].

\subsection{Spinal curvatures examination}

Spinal posture was assessed using the fourth-generation Moiré apparatus (CQ Elektronik System, Wrocław, Poland) which maps the anteroposterior curvatures based on the direct visual assessment of participants with a camera. All measurements were performed by the same investigator at the same time of day (morning) and under similar conditions (dark room with controlled ambient temperature). The examination took place in several stages. First, the spinous processes of the $\mathrm{C} 7-\mathrm{S} 1$ vertebrae were marked along the spine of each participant. Then, participants were positioned parallel to the camera at a distance of $2.6 \mathrm{~m}$ with their back facing the camcorder, maintaining a natural posture and looking straight ahead, with their upper limbs hanging down freely beside their trunk, lower limbs upright in the knee joints with the same distribution of body weight, feet parallel to each other and heels in a line. The images of each participant were saved.

Sagittal spinal curvature was assessed by evaluating the following angular parameters of the spine [3], [11]: alpha angle $\left[^{\circ}\right]$, representing the inclination of the lumbosacral section; beta angle $\left[{ }^{\circ}\right]$, the inclination of the thoracolumbar section; and gamma angle $\left[{ }^{\circ}\right]$, the inclination of the upper thoracic section (Fig. 1).

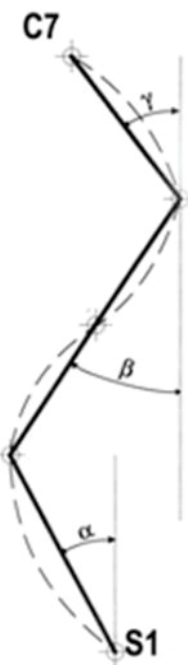

Fig. 1. Angular parameters of the sagittal spinal curvatures; $\alpha$-alfa angle (lumbosacral angle), $\beta$ - beta angle

(thoracolumbar angle), $\gamma$ - gamma angle (upper thoracic angle), C7 - seventh cervical vertebra, S1 - first sacral vertebra

\subsection{Training intervention}

The training intervention was based on previous guidelines for cancer survivors [20]. The exercise programmes consisted of 45-min sessions, performed two times a week over 8 weeks under the supervision of a specially trained instructor.

In the SG, 8 weeks of NW training was implemented, while a standard general exercise programme was performed by participants in the CG. Exercise programmes were organised as individual training units that were similar for all regards. The initial and final parts of every unit were divided equally in terms of duration and types of exercises applied.

For $45-50 \%$ of each 45 -min exercise session, the training intensity was kept below $70 \%$ of the participant's maximum heart rate based on their age. Heart pulsometers monitored the participant's heart rate at least twice during each training unit and the exercise intensity was found to be within the desired limits. 
The intensity of training interventions in both the SG and CG was increased by gradually reducing the number of rest breaks. The number of rest breaks, including upper limb relaxation exercises and respiratory exercises, was reduced every second week from five breaks (weeks 1-2) to two breaks (weeks 7-8). At the same time, the distance covered in the SG was increased from $2400 \mathrm{~m}$ (weeks 1-2) to $3600 \mathrm{~m}$ (weeks $7-8$ ). For the CG, the number of repetitions for each set of exercises was increased from 10 (weeks 1-2) to 16 repetitions (weeks $7-8$ ).

For both groups, an additional criterion for inclusion in the study was an attendance rate of at least $80 \%$.

\subsection{Statistical analysis}

Statistical analyses were performed using Microsoft Excel and Statistica 13.0 (StatSoft). Normality of the raw data was verified using the Shapiro-Wilk test, which confirmed that the data were normally distributed.

\section{Results}

\section{Effect of Nordic walking on strength-velocity parameters of the trunk muscles}

The two-way ANOVA revealed significant group $x$ time interactions for the main outcomes of the study, the TW of the trunk flexor muscles and AP of the trunk extensor and flexor muscles (Table 3 ).

Between-group comparisons: The Scheffe post hoc test revealed statistically significant differences $(p<0.01)$ in TW and AP between the groups following the training interventions, irrespective of the muscle group under investigation. The NW group had greater values of strength-velocity parameters of the trunk muscles compared to the CG. There were no significant differences between the groups in any examined parameters prior to the training intervention.

Within-group comparisons: For the NW group, statistically significant differences $(p<0.01)$ were found between the pre- and post-training intervention values. The post-training values of TW and AP in the

Table 3. Changes in strength-velocity parameters of the trunk muscles pre- and post-training intervention

\begin{tabular}{|c|c|c|c|c|c|c|}
\hline \multirow{2}{*}{\multicolumn{2}{|c|}{ Parameters }} & \multirow{2}{*}{ Group } & PRE- & POST- & \multirow{2}{*}{$F$} & \multirow{2}{*}{$p$} \\
\hline & & & \multicolumn{2}{|c|}{ training intervention } & & \\
\hline \multirow{4}{*}{$\begin{array}{l}\text { Total work } \\
\text { at } 120^{\circ} / \mathrm{s} \\
{[\mathrm{J}]}\end{array}$} & \multirow{2}{*}{$\mathrm{E}$} & $\mathrm{SG}$ & $306.99 \pm 347.72$ & $525.29 \pm 371.59$ & \multirow{2}{*}{3.68} & \multirow{2}{*}{0.06} \\
\hline & & $\mathrm{CG}$ & $187.78 \pm 144.08$ & $232.14 \pm 249.70$ & & \\
\hline & \multirow{2}{*}{$\mathrm{F}$} & $\mathrm{SG}$ & $258.40 \pm 206.27$ & $440.76 \pm 251.66$ & \multirow{2}{*}{6.71} & \multirow{2}{*}{$0.01 *$} \\
\hline & & $\mathrm{CG}$ & $184.18 \pm 105.15$ & $235.96 \pm 158.62$ & & \\
\hline \multirow{4}{*}{$\begin{array}{l}\text { Average } \\
\text { power } \\
\text { at } 120 \% \text { s } \\
{[\mathrm{W}]}\end{array}$} & \multirow{2}{*}{$\mathrm{E}$} & SG & $16.74 \pm 17.53$ & $31.66 \pm 24.19$ & \multirow{2}{*}{5.02} & \multirow{2}{*}{$0.03 *$} \\
\hline & & $\mathrm{CG}$ & $10.07 \pm 8.53$ & $12.53 \pm 13.27$ & & \\
\hline & \multirow{2}{*}{$\mathrm{F}$} & $\mathrm{SG}$ & $14.37 \pm 11.38$ & $25.29 \pm 6.74$ & \multirow{2}{*}{7.55} & \multirow{2}{*}{$0.01 *$} \\
\hline & & $\mathrm{CG}$ & $9.91 \pm 14.63$ & $12.68 \pm 9.54$ & & \\
\hline
\end{tabular}

$\mathrm{E}$ - trunk extensors, $\mathrm{F}$ - trunk flexors, $\mathrm{SG}-$ study group, $\mathrm{CG}-$ control group, $\mathrm{F}-$ test value, *significant group $\times$ time interaction (two-way ANOVA, $p<0.05$ ).

Pearson's chi-squared test was performed to analyse discrete properties relating to cancer treatment within the two groups. Anthropometric characteristics were compared using Student's $t$-test. Within- and between-group comparisons were performed by twoway ANOVA with Scheffe post hoc tests to examine the effects of the interventions on isokinetic trunk muscle endurance and sagittal spinal curvatures.

Pearson's correlation coefficients were calculated to describe the strength and direction of the correlation between trunk muscle function and sagittal spinal curvatures within the two groups.

All statistical tests and coefficients were considered significant at $p<0.05$.
SG, irrespective of the muscle group under investigation, were higher than the pre-training values. There were no significant changes in the Scheffe post hoc test for any of the examined parameters in the CG.

Pre- and post-training intervention values are expressed as mean \pm standard deviation.

\section{Effect of Nordic walking on body posture parameters}

There were no statistically significant group $\times$ time interactions $(p<0.05)$ for the anterior-posterior curvatures of the spine (Table 4). Similarly, no significant differences $(p>0.05)$ were detected in the post 
hoc analyses of spinal curvatures parameters in the SG and CG. No changes in the analysed parameters were observed between SG and CG or within each group for the pre- and post-training intervention values.

Table 4. Changes in body posture parameters pre- and post-training intervention

\begin{tabular}{|c|c|c|c|c|c|}
\hline \multirow{2}{*}{ Parameters } & \multirow{2}{*}{ Group } & Pre- & Post- & \multirow{2}{*}{$F$} & \multirow{2}{*}{$p$} \\
\hline & & \multicolumn{2}{|c|}{ training intervention } & & \\
\hline \multirow{2}{*}{$\alpha\left[^{\circ}\right]$} & SG & $16.35 \pm 4.36$ & $13.96 \pm 5.35$ & \multirow{2}{*}{1.88} & \multirow{2}{*}{0.18} \\
\hline & $\mathrm{CG}$ & $16.04 \pm 5.76$ & $15.77 \pm 6.14$ & & \\
\hline \multirow{2}{*}{$\beta\left[^{\circ}\right]$} & SG & $12.64 \pm 4.60$ & $11.02 \pm 3.60$ & \multirow{2}{*}{1.37} & \multirow{2}{*}{0.25} \\
\hline & $\mathrm{CG}$ & $11.80 \pm 4.17$ & $11.26 \pm 4.82$ & & \\
\hline \multirow{2}{*}{$\gamma\left[^{\circ}\right]$} & SG & $16.97 \pm 3.05$ & $15.72 \pm 3.86$ & \multirow{2}{*}{1.54} & \multirow{2}{*}{0.22} \\
\hline & $\mathrm{CG}$ & $18.22 \pm 3.94$ & $18.08 \pm 4.02$ & & \\
\hline
\end{tabular}

$\mathrm{SG}$ - study group, $\mathrm{CG}$ - control group, $\alpha$ - lumbosacral angle, $\beta$ - thoracolumbar angle, $\gamma$ - upper thoracic angle, $F$ - test value, *significant group $\times$ time interaction (two-way ANOVA, $p<0.05)$.

Pre- and post-training intervention values are expressed as mean \pm standard deviation.

\section{Correlation between trunk muscle endurance and sagittal spinal curvatures}

Pearson's correlation coefficients were calculated, but no statistically significant $(p>0.05)$ correlations of functional-postural dependencies of the trunk muscles were found prior to the training intervention (Table 5).

A comparison of the trunk muscle function for the sagittal spinal curvatures after the training intervention for both groups combined (SG + CG, $n=39$ ) showed a statistically significant tendency $(p<0.05)$ toward reduced inclination of the upper thoracic section (gamma), together with an increase in all examined functional parameters of flexor and extensor muscles of the trunk ( $r=-0.33$ to $r=-0.37)$.

When the two training modalities were assessed separately, no statistically significant $(p>0.05)$ relationships for functional-postural dependencies of the trunk muscles were observed (Table 6).

\section{Discussion}

Trunk muscles are fundamental for the dynamic balance of the body and ensuring proper stability of the spine. Modulating the activity and function of these muscles according to the task is critical for locomotion. During walking, the trunk extensor muscles maintain balance, which is disrupted by arm swinging, and support pelvis movement. In turn, the flexor muscles change their activation pattern from tonic to phasic in a velocitydependent manner to stabilise and mobilise gait [23].

Table 5. Pearson's correlation coefficient of functional-postural dependencies of the trunk muscles before the training intervention

\begin{tabular}{|c|c|c|c|c|c|c|c|c|c|c|}
\hline & \multicolumn{3}{|c|}{$\begin{array}{c}\mathrm{NW}+\mathrm{G} \\
\text { All patients }(n=39)\end{array}$} & \multicolumn{3}{|c|}{$\begin{array}{c}\text { NW } \\
\text { SG }(n=19)\end{array}$} & \multicolumn{3}{|c|}{$\begin{array}{c}\mathrm{G} \\
\mathrm{CG}(n=20)\end{array}$} \\
\hline & & $\alpha$ & $\beta$ & $\gamma$ & $\alpha$ & $\beta$ & $\gamma$ & $\alpha$ & $\beta$ & $\gamma$ \\
\hline \multirow{2}{*}{ TW } & $\mathrm{E}$ & 0.10 & 0.03 & -0.12 & 0.21 & -0.02 & 0.04 & -0.05 & 0.10 & -0.34 \\
\hline & $F$ & 0.09 & 0.00 & -0.12 & 0.26 & -0.09 & 0.01 & -0.14 & 0.12 & -0.24 \\
\hline \multirow{2}{*}{ AP } & $\mathrm{E}$ & 0.07 & -0.04 & -0.16 & 0.19 & -0.13 & -0.03 & -0.10 & 0.05 & -0.31 \\
\hline & $\mathrm{F}$ & 0.03 & -0.12 & -0.14 & 0.22 & -0.27 & -0.09 & -0.21 & 0.06 & -0.14 \\
\hline
\end{tabular}

NW - Nordic walking, G - general exercises, TW - total work, AP - average power, E - trunk extensors, F - trunk flexors, $\alpha$ - lumbosacral angle, $\beta$-thoracolumbar angle, $\gamma$ - upper thoracic angle, $*$ denotes statistical significance at $p<0.05$.

Table 6. Pearson's correlation coefficient of functional-postural dependencies of the trunk muscles after the training intervention

\begin{tabular}{|c|c|c|c|c|c|c|c|c|c|c|}
\hline & \multicolumn{3}{|c|}{$\begin{array}{c}\mathrm{NW}+\mathrm{G} \\
\text { All patients }(n=39)\end{array}$} & \multicolumn{3}{|c|}{$\begin{array}{c}\mathrm{NW} \\
\mathrm{SG}(n=19)\end{array}$} & \multicolumn{3}{|c|}{$\begin{array}{c}\mathrm{G} \\
\mathrm{CG}(n=20)\end{array}$} \\
\hline & & $\alpha$ & $\beta$ & $\gamma$ & $\alpha$ & $\beta$ & $\gamma$ & $\alpha$ & $\beta$ & $\gamma$ \\
\hline \multirow{2}{*}{ TW } & $\mathrm{E}$ & -0.01 & -0.17 & $-0.34 *$ & 0.39 & -0.20 & -0.31 & -0.35 & -0.18 & -0.16 \\
\hline & $\mathrm{F}$ & 0.01 & -0.19 & $-0.33 *$ & 0.30 & -0.25 & -0.27 & -0.18 & -0.16 & -0.19 \\
\hline \multirow{2}{*}{ AP } & $E$ & 0.02 & -0.15 & $-0.37^{*}$ & 0.38 & -0.18 & -0.31 & -0.31 & -0.16 & -0.27 \\
\hline & $\mathrm{F}$ & 0.01 & -0.20 & $-0.34 *$ & 0.29 & -0.31 & -0.25 & -0.13 & -0.13 & -0.26 \\
\hline
\end{tabular}

NW - Nordic walking, $\mathrm{G}$ - general exercises, TW - total work, AP - average power, E - trunk extensors, $\mathrm{F}$ - trunk flexors, $\alpha$ - lumbosacral angle, $\beta$-thoracolumbar angle, $\gamma$ - upper thoracic angle, $*$ Denotes statistical significance at $p<0.05$. 
Nordic walking is a safe and simple form of exercise that has become popular in recent years, which helps to promote a normal gait pattern in older adults [5]. The inclusion of walking poles causes prolongation of the step length, trunk muscle activation and coactivation of the spinal flexors and extensors [4]-[6], [17], [22], which, in turn, increases trunk muscle activity. Previous findings based on surface electromyography (EMG) revealed that the alternating pushing action of the poles in accordance with the technique generates greater torque along the longitudinal axis of the body. It is believed that elevated rectus abdominis activity associated with NW may be required to counteract the forces generated by the poles on the ground. Moreover, extending the step length by practicing NW results in greater trunk rotation, which requires increased external oblique activity. Therefore, NW requires enhanced activity of the abdominal muscles [22], [23]. Accordingly, in response to the $\mathrm{NW}$ training intervention, the $\mathrm{SG}$ showed significant increases in AP and TW of the trunk flexor muscles. Similar changes did not occur in the CG in response to the general exercise programme.

A combined analysis (Teager-Kaiser energy, statistical non-parametric mapping) employed by Zoffoli et al. [22] showed comparable involvement of the spine extensors during natural walking and NW. This suggests that NW it a better balance exercise for the trunk muscles than natural walking. However, this is only the case for healthy individuals. In women who have undergone $\mathrm{BC}$ treatment, a stabilometry evaluation showed imbalances, with more energy expenditure required to maintain postural balance [13]. The erector spinae longissimus and multifidus muscles are considered critical to support the upper body when the foot hits the ground during walking [22]. This explains why we observed a statistically significant increase in AP and TW for extensor muscles in response to NW in the current study.

It is believed that the increased trunk muscle activity during NW contributes to improved trunk control and spinal stability [23]. In reference to the functional role of trunk muscles during walking and in maintaining body posture, the findings of our research suggest that assessment of the impact of NW on the shape of the anteroposterior spine curvatures in women following $\mathrm{BC}$ treatment is of interest. Disorders arising as adverse side effects of $\mathrm{BC}$ treatment can affect the musculoskeletal and nervous systems, and impact the functioning of the posture regulation system. Over $80 \%$ of women treated for BC have faulty body posture. Limited joint mobility, contrac- tures, pain, muscle weakness, tissue fibrosis and peripheral nerve damage, together with psychological disorders, are the main causes of adverse changes in body posture and trunk statics of women treated for $\mathrm{BC}$ [13], [14]. NW is a full-body exercise that strengthens the paraspinal muscles, so it might be useful for recovering the normal angle balance of the trunk. After 12 weeks of NW training in elderly women, the muscles around the spine were found to be better aligned and the left-right balance was in equilibrium, which is likely due to the simultaneous involvement and cooperation of abdominal muscles, pelvic muscles and sensory nervous system [16]. However, scientific reports investigating the impact of NW on body posture are limited. Dalton and Nantel [5] stated that claims of improved postural alignment appear to be entirely unsupported by scientific research up to this point. Furthermore, one-off assessment of gait and posture with accelerometery systems and three-dimensional motion capture systems showed no changes in posture. Learning the NW technique then immediately performing laboratory testing may not be an entirely accurate assessment. This NW training study was continued for a period of 8 weeks. Dalton and Nantel [4] found an improvement in the postural alignment of the trunk in the sagittal direction after the 8-week NW intervention. This was observed solely during the $5-\mathrm{m}$ walk trials, with no change in the 6-min walk test. However, due to factors including fatigue and insufficient attention to the poling technique, maintaining this improved posture over a longer period of time could be difficult. The authors [4] speculated that proper acquisition of the skill and a more rigorous intervention programme would lead to postural improvements over a longer duration, such as during the 6-min walk test. However, in our research, no statistically significant postural changes were observed in response to NW.

It is worthy of a note that maintaining the trunk orientation during NW requires synergy of the trunk extensor and flexor muscles [22]. A decrease in lumbar muscle strength reduces the stability of the spine during posture control activities like walking, which leads to an imbalance in lumbar extensor and flexor strength. In addition, maintaining an upright posture during locomotion increases the tension of the transverse abdominis muscles [9], [19].

In the current study, no statistically significant changes were found in the functional-postural condition before and after the NW and general exercise interventions; however, when the groups were combined (Nordic walking + general exercise, $n=39$ ), statistically significant correlations were observed. This find- 
ing suggests that increased trunk muscle function promotes a reduction in thoracic kyphosis, which is particularly beneficial in women treated for $\mathrm{BC}$, as this group has a particular tendency for posture kyphosis [2], [10], [11]. This is confirmed by the significant relationships between the decreased upper thoracic angle and increased TW and AP of the trunk flexors and extensors after the training interventions. It is believed that in order to assess the functional and postural relationships of the trunk muscles in response to a varied physical activity programme, a larger number of participants is required. Based on the research undertaken in the current study, we can thus confirm functional-postural correlations that occurred in response to physical activity overall

\section{Conclusions}

Nordic walking leads to a statistically significant increase in trunk muscle function compared to a standard general exercise programme. However, correction of the body posture of women treated for $\mathrm{BC}$ was not achieved after the 8-week NW or general exercise interventions. When the groups were combined, a functional-postural correlation was observed after the intervention. Therefore, correction of the body posture of women treated for BC is possible, but to observe a significant effect, studies must include a greater number of participants taking part in the physical activity.

Our results should encourage physicians to consider personalised rehabilitation programmes of women after $\mathrm{BC}$ treatment with a particular focus on postural disorders. These rehabilitation programmes should not only focus on the recovery of upper limb function, but also include exercises to improve trunk muscle function and postural control.

\section{References}

[1] ARUin A.S., LATASH M.L., Directional specificity of postural muscles in feed-forward postural reactions during fast voluntary arm movements, Exp. Brain. Res., 1995, 103 (2), $323-332$

[2] Barbosa A.J., Amorim M.H., Zandonade E., Delaprane M.L., Evaluation of body posture in women with breast cancer, Rev. Bras. Ginecol. Obstet., 2013, 35(5), DOI: 10.1590/S0100-72032013000500005.

[3] Barczyk-Pawelec K., DziUbeK W., Piechura J.R., RożeK K., Correlations between somatic features, anteroposterior spinal curvatures and trunk muscle strength in schoolchildren, Acta Bioeng. Biomech., 2017, 19(1), DOI: 10.5277/ABB-00537-2015-03.
[4] Dalton C., NANTel J., Nordic walking improves postural alignment and leads to a more normal gait pattern following weeks of training: A pilot study, J. Aging Phys. Act., 2016, 24 (4), DOI: 10.1123/japa.2015-0204

[5] Dalton C.M., NANTEl J., Substantiating Appropriate Motion Capture Techniques for the Assessment of Nordic Walking Gait and Posture in Older Adults, J. Vis. Exp., 2016, 111, DOI: $10.3791 / 53926$.

[6] DZIUBA A.K., ŻUREK G., GARRARD I., WierZBicKA-DAMSKA I., Biomechanical parameters in lower limbs during natural walking and Nordic walking at different speeds, Acta Bioeng. Biomech., 2015, 17 (1), DOI: 10.5277/ABB-00077-2014-01.

[7] Hojan K., Manikowska F., Molinska-Glura M., Chen P.J.B., JOZWIAK M., The impact of an external breast prosthesis on the gait parameters of women after mastectomy, Cancer Nursing, 2014, 37 (2), DOI:10.1097/NCC.0b013e3182919576.

[8] JÖnsson C., Johansson K., Pole walking for patients with breast cancer-related arm lymphedema, Physiother. Theory Pract., 2009, 25 (3), DOI: 10.1080/09593980902776621.

[9] KIM H. M., The effect of postural walking exercise on back pain and recovery of trunk balance among patients with low back pain, Physical Education major Graduate School of Education Sogang University, 2011.

[10] Elsayed W., Farrag A., Muaidi Q., Almulhim N., Relationship between sagittal spinal curves geometry and isokinetic trunk muscle strength in adults, Eur. Spine J., 2018, 27 (8), DOI: $10.1007 / \mathrm{s} 00586-017-5454-3$.

[11] Malicka I., HanUSZKiewicz J., StefańsKa M., BarcZyK K., WOŹNIEWSKI M., Relation between trunk muscle activity and posture type in women following treatment for breast cancer, J. Back Musculoskelet. Rehabil., 2010, 23 (1), DOI: 10.3233/ BMR-2010-0242

[12] Malicka I., Stefańska M., RudziaK M., JaRmoluk P., PAWŁOWSKA K., SZCZEPAŃSKa-GIERACHA J., WoŹNIEWSKI M., The influence of Nordic walking exercise on upper extremity strength and the volume of lymphoedema in women following breast cancer treatment, Isokinet. Exerc. Sci., 2011, 19 (4), DOI 10.3233/IES-2011-0430.

[13] Mangone M., Bernetti A., Agostini F., Paoloni M., De Cicco F.A., Capobianco S.V., Paolucci T., Changes in spine alignment and postural balance after breast cancer surgery: a rehabilitative point of view, Biores. Open Access, 2019, 8 (1), DOI: 10.1089/biores.2018.0045.

[14] Montezuma T., DE OliveIra Guirro E.C., Leite M.M.D.O.L., VERNAL S., Changes in postural control in mastectomized women, J. Cancer Ther., 2014, 5 (6), DOI: 10.4236/jct.2014.56056.

[15] Morsø L., Hartvigsen J., Puggaard L., Manniche C., Nordic Walking and chronic low back pain: design of a randomized clinical trial, BMC Musculoskelet. Disord., 2006, 7 (1), DOI: 10.1186/1471-2474-7-77

[16] PARK H.S., Lee S.N., Sung D.H., ChOI H.S., Kwon T.D., PARK G.D., The effect of power Nordic walking on spine deformation and visual analog pain scale in elderly women with low back pain, J. Phys. Ther. Sci., 2014, 26 (11), DOI: 10.1589/jpts.26.1809.

[17] Pellegrini B., Peyré-Tartaruga L.A., Zoppirolli C., Bortolan L., Savoldelli A., Minetti A.E., Schena F., Mechanical energy patterns in nordic walking: comparisons with conventional walking, Gait Posture, 2017, 51, DOI: 10.1016/j.gaitpost.2016.10.010.

[18] RABElo M., FACHIN-MARTINS E., Inter-rater and test/retest reliabilities of the isokinetic measurements: assessing strength and endurance of the trunk muscles in two different pro- 
tocols for able-bodied and post-stroke hemiparesis, Top Stroke Rehabil., 2018, 25 (26), DOI: 10.1080/ 10749357.2018.1481568.

[19] Reuter I., Mehnert S., Leone P., Kaps M., Oechsner M., ENGELHARDT M., Effects of a flexibility and relaxation programme, walking, and nordic walking on Parkinson's disease, J. Aging Res., 2011, DOI: 10.4061/2011/232473.

[20] Schmitz K.H., Courneya K.S., Matthews C., Demark-Wahnefried W., Galvão D.A., et al., American College of Sports Medicine roundtable on exercise guidelines for cancer survivors, Med. Sci. Sports Exerc., 2010, 42 (7), DOI: 10.1249/ MSS.0b013e3181e0c112.
[21] Sprod L.K., Drum S.N., Bentz A.T., Carter S.D., SCHNEIDER C.M., The effects of walking poles on shoulder function in breast cancer survivors, Integr. Cancer Ther., 2005, 4 (4), DOI: 10.1177/1534735405282212.

[22] Zoffoli L., Ditroilo M., Federici A., LuCERTINI F., Patterns of trunk muscle activation during walking and pole walking using statistical non-parametric mapping, J. Electromyogr. Kinesiol., 2017, 37, DOI: 10.1016/j.jelekin.2017.09.002.

[23] Zoffoli L., Lucertini F., Federici A., Ditroilo M., Trunk muscles activation during pole walking vs. walking performed at different speeds and grades, Gait Posture, 2016, 46, DOI: 10.1016/j.gaitpost.2016.02.015. 